\title{
Quadratus Lumborum Block for Back Pain Related to Pancreatic Cancer - A Report of Two Cases
}

\author{
Uri Hochberg, MD ${ }^{1,2}$, Amir Minerbi, MD, $\mathrm{PhD}^{3}$, Rodrigo Diez Tafur, $\mathrm{MD}^{4}$, and Jordi Perez, MD, PhD
}

Pancreatic cancer is often accompanied by severe pain. Patients typically experience upper abdominal and/or thoracolumbar back pain. For those cases failing to respond to standard medical management, as suggested by the World Health Organization, interventions designated at interruption of the sympathetic axis (such as neurolysis of the celiac plexus or splanchnic nerves) have been shown to be efficacious. Other than axial drug delivery, there are few interventional alternatives in patients with pancreatic cancerrelated pain.

There is little knowledge regarding the therapeutic effects of treating peripheral somatic soft tissue among oncological patients. Here we report on 2 such patients, whose back pain improved following a quadratus lumborum block.

Two patients diagnosed with pancreatic cancer presented with severe back pain. The pain pattern and patients' physical exams were compatible with myofascial pain arising from the quadratus lumborum muscle, possibly irritated by the abdominal tumor. Advanced pain management, including long- and short-acting opioids and adjuvants, as well as celiac plexus neurolytic block, failed to provide satisfactory pain relief. Given the apparent muscular origin of the pain, a bilateral ultrasound-guided quadratus lumborum block was performed. Four weeks post procedure, the 2 patients reported substantial pain relief supported by reduced consumption of pain medication and improved functional status. No adverse events or complications were observed in either case.

In the patients described here, quadratus lumborum block proved to be safe and efficacious in alleviating back pain related to pancreatic cancer. In our opinion, clinicians should be aware of the possible contribution of a myofascial component to pain in pancreatic cancer and in cancer-related pain in general.

Key words: Quadratus lumborum block, cancer pain, pancreatic cancer, pain control, myofascial pain syndrome, interventional pain management
Pancreatic cancer is often accompanied by severe pain typically reported over the upper abdominal and/ or mid-back areas. This pain may be a consequence of several possible mechanisms including direct

From : ${ }^{1}$ McGill University, Quebec, Canada; ${ }^{2}$ Sackler School of Medicine, Tel Aviv University, Tel Aviv, Israel; ${ }^{3}$ Institute for Pain Medicine, Rambam Health Care Campus, Haifa, Israel; ${ }^{4}$ Alan Edwards Pain Management Unit, McGill University Health Centre, Montreal, Quebec, Canada; ${ }^{5}$ Cancer Pain Clinic, Division of Supportive and Palliative Care, McGill University Health Centre, Montreal, Quebec, Canada

Author for correspondence: Uri Hochberg, MD

Address: Chronic and acute pain center, Tel Aviv Sourasky Medical Center, 6 Weizmann Street. Tel Aviv 6423906, Israel

E-mail: urihochberg@hotmail.com pressure by the tumor, infiltration of neural structures, neurogenic inflammation, and distant metastatic spread (1). These mechanisms could manifest as neuropathic or nociceptive, visceral, or somatic pain. In addition, myofascial pain sometimes accompanies some tumors, with or without direct invasion of the muscles (2). Myofascial pain syndrome may develop abruptly or gradually, and may not respond to treatments that are effective for other sources of pain. As an example, opioids, which are highly effective for treating most types of cancer-related pain, are often ineffective in relieving myofascial pain (3). Furthermore, myofascial pain may persist even after successful neurolytic blocks, targeting the visceral component of pancreatic cancer. This pain, arising in the quadratus lumborum, psoas, and/or erector 
spinae is possibly maintained due to the persistent visceral-somatic convergence (4).

Management of pain accompanying pancreatic cancer is often multimodal, and includes, among other measures, pharmacotherapy, invasive procedures, and psychosocial support. Pharmacologic analgesic management of pancreatic cancer-related pain does not always provide satisfactory pain relief; interventional modalities such as interruption of the sympathetic axis (celiac plexus or splanchnic nerve neurolysis) have been shown efficacious for both the neuropathic and the visceral components of pain (5). Different pain sources may respond differentially to specific treatment modalities. As an example, myofascial pain is often resistant to opioids, and may persist after other pain sources have been successfully treated (6).

The quadratus lumborum (QL) muscle is a posterior abdominal wall structure that is engulfed by the thoracolumbar fascia (TLF), which in turn is comprised of a few fascia and aponeurosis sheets dividing the posterior abdominal wall muscles from the para-spinal muscles (7). Typically, myofascial pain arising from the QL muscle is triggered or worsened by activities like walking, sitting, and changing posture. On physical examination, pain can be reproduced by palpation below the rib line and lateral to the transverse process of $L 1$ and over the lateral rim of the iliac crest. Active stretching and lateral lumbar flexion also typically reproduce the pain (8).

A QL block was first described as a variation of the transversus abdominis plane block. It was developed to provide more reliable analgesia cephalad to the umbilicus. To date, 4 ultrasound (US)-guided QL blocks have been described in the literature, differing by the final position of the needle tip with respect to the QL muscle. Type $1 \mathrm{QL}$ block is performed with the needle positioned lateral to the muscle; type 2 with the needle positioned at the posterior, more superficial, surface of the muscle; and types 3 and 4 are intramuscular and transmuscular blocks, respectively (Fig. 1) (9).

Here we describe 2 pancreatic cancer patients, whose pain was predominantly myofascial and who

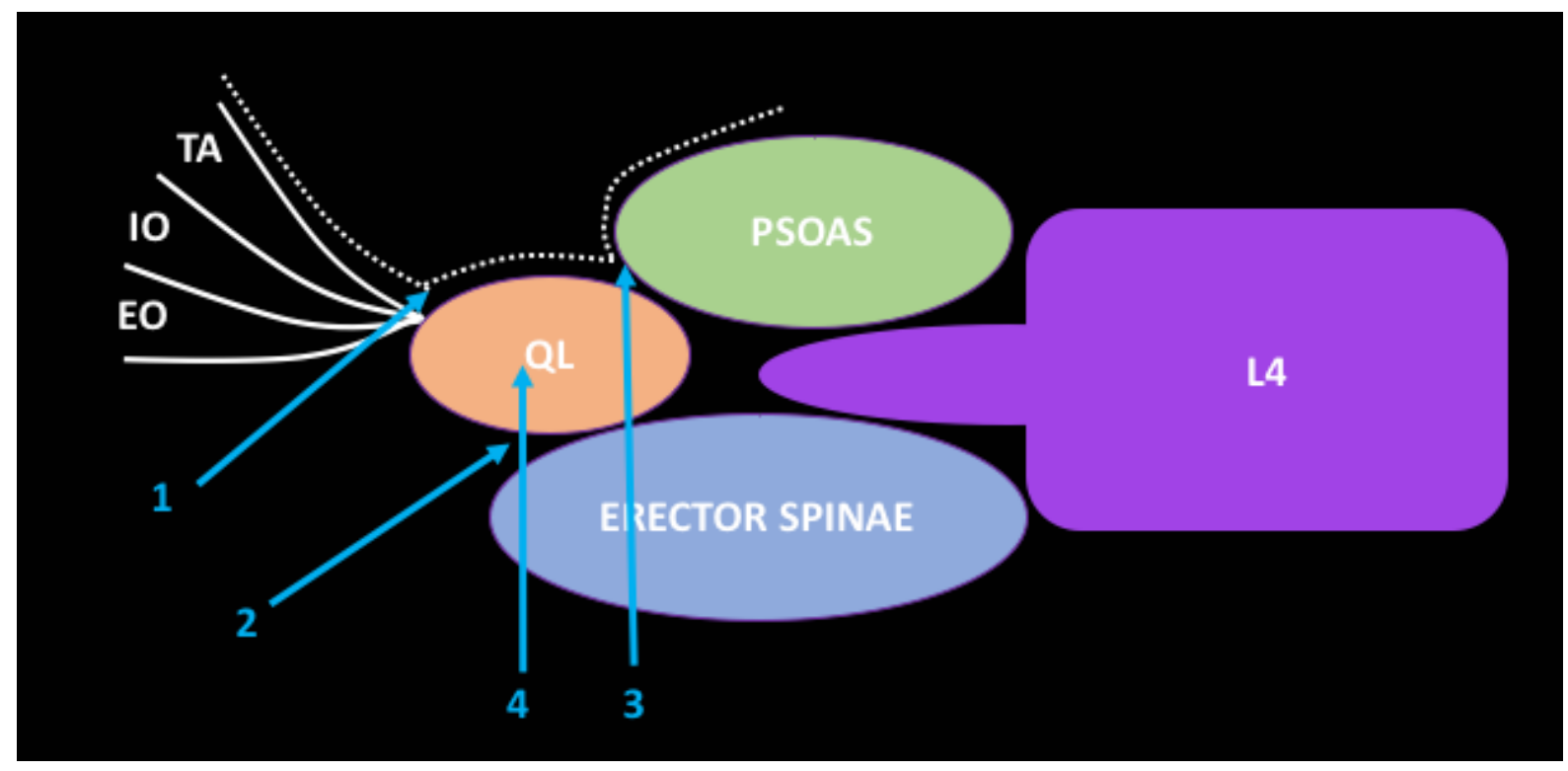

Fig. 1. An illustration demonstrating the position of the needle tip in the 4 commonly used techniques for performing the quadratus lumborum (QL) block. The patients described here underwent QL block type 2. 
did not respond to standard pharmacotherapy or to celiac plexus neurolysis. Interestingly, their pain improved significantly following a US-guided QL block.

\section{CASE REPORTS}

Case 1: The first case involved a 47-year-old female patient diagnosed with locally advanced pancreatic cancer. The patient was receiving systemic neoadjuvant chemotherapy with the hope that the response would deem the cancer operable. The patient had had a biliary drain placed. She had been referred to the cancer pain clinic for the treatment of constant and debilitating abdominal pain accompanied by midthoracic and lumbar pain. Pain was described as sharp and aggravated by movements of the trunk and turning in bed. Pain was not associated with radiation to the lower extremities, motor weakness, or saddle anesthesia.

On physical examination, the patient presented with antalgic gait, severe tenderness and tightness of lumbar spine erector muscles, and sensitivity to light pressure along these muscles. Lumbar spine ranges of motion were restricted, triggering the patient's typical pain on lateral flexion and rotation. A computed tomographic scan of the lumbar spine was normal.

A combination of long- and short-acting hydromorphone and adjuvant acetaminophen was prescribed with little effect. The patient subsequently received a celiac plexus neurolysis without a satisfactory analgesic effect. Given the poor analgesic effects of the conservative and invasive interventions, as well as the characteristics of the patient's pain and the findings on physical examination, myofascial pain of both QL muscles was suspected. The patient subsequently underwent a bilateral US-guided QL block.

One week after the procedure, the patient reported satisfactory control of the back and abdominal pain, which had decreased from 6 to 3 (on a 0-10 numeric rating scale), significantly improved daily function, and had stopped taking any opioids. The analgesic effect was still present 3 months later.

Case 2: The second case involved a 79-year-old female patient diagnosed with locally advanced adenocarcinoma of the head of the pancreas with metastases to the liver and both lungs. The patient had undergone biliary stenting and received chemotherapy and anticoagulation for deep venous thrombosis. The patient was referred to the cancer pain clinic for the treatment of back pain, at times radiating anteriorly to the lower abdomen. Pain was described as constantly present in the lumbar and lower thoracic areas. It was aggravated by physical activity, lateral spine flexion, and deep breathing. Pain limited the patient's functional capacity and had a negative effect on her mood. The physical examination demonstrated restricted range of motion of the lumbar spine, with reproduction of the pain on rotation and lateral bending and palpation over the iliac crest.

The patient was prescribed a combination of longand short-acting hydromorphone and pregabalin with little effect on her pain. The patient subsequently underwent celiac plexus neurolysis, which failed to provide significant pain relief. With the hypothesis of myofascial pain syndrome (MPS) of the QL, the patient received a bilateral US-guided QL injection. Immediately after the block, the patient reported mild pain relief. In a follow-up visit 4 weeks later, the patient reported a resolution of her back pain and a significant improvement in the severity of her abdominal pain, which had decreased from 7 to 4 (on a 0-10 numeric rating scale) and was described as intermittent and bearable. This was accompanied by substantial improvement in the patient's daily functioning, including house chores and cooking. Pain medications remained unchanged after the procedure.

\section{PROCEDURES}

QL blocks were performed in the office with a US machine (Zonare, Shenzhen, China) and a 6-2 curve array transducer. Patients were placed prone and the lower lumbar area was prepped with $0.5 \%$ chlorhexidine on each side at a time. A $3 \frac{1}{2}$-inch, 25 -gauge spinal needle was inserted using the in-plane approach with the needle tip placed at the posterior aspect of the QL muscle, corresponding with a QL type 2 block (Figs. 1-3). Under US control, a hydro-dissection of the muscle planes was observed along with accumulation and spread of the injectate between the medial leaflet of the TLF and the QL muscle. Twenty $\mathrm{mL}$ of $0.5 \%$ lidocaine was injected in each site (9). This volume of injectate is 


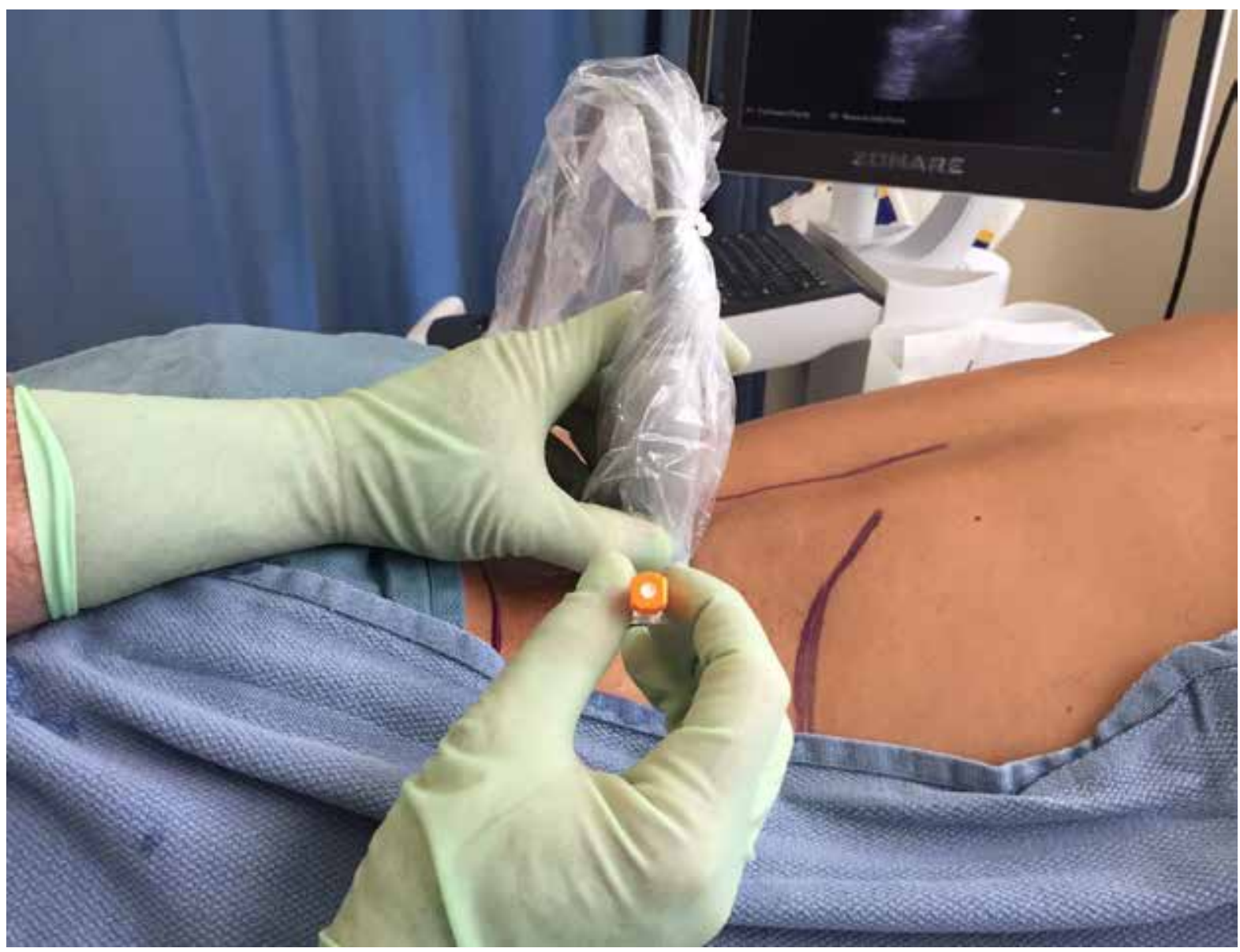

Fig. 2. Ultrasound-guided quadratus lumborum block. The following surface anatomy structures are marked on the skin: midline longitudinal line — lumbar spine midline; curved superior line - lower rib line; inferior line—iliac crest.

like the volumes used in other interfascial blocks and was chosen based on previous reports demonstrating that using this volume allows the injectate to spread to the lower thoracic levels (10). After the procedure, patients were monitored for 30 minutes to rule out postprocedural complications. All patients were discharged with specific instructions to perform stretching exercises recommended for this muscle.

\section{DISCUSSION}

The 2 patients described here presented with disabling pain accompanying pancreatic cancer; their pain had been unresponsive to conventional pharmacotherapy and to celiac plexus blockade. Some characteristics of the pain, including certain findings on physical examination, led us to suspect the QL muscles as a possible origin for some of the pain. Performing a QL block in these patients resulted in substantial pain relief.

In patients suffering from pancreatic cancer, the important visceral nociception traveling from T912 causes spinal cord central sensitization, which overlaps not only in the innervation of the abdominal muscles, but also in that of the back muscles, and in particular, the QL. Even when the visceral component of pain is successfully disrupted, the muscles often remain a source of ongoing pain.

Pain relief in response to QL block, but not following administration of opioids and blockade of the celiac 
Fig. 3. An ultrasound image as seen during type 2 quadratus lumborum block (a). The surrounding structures are highlighted in (b).
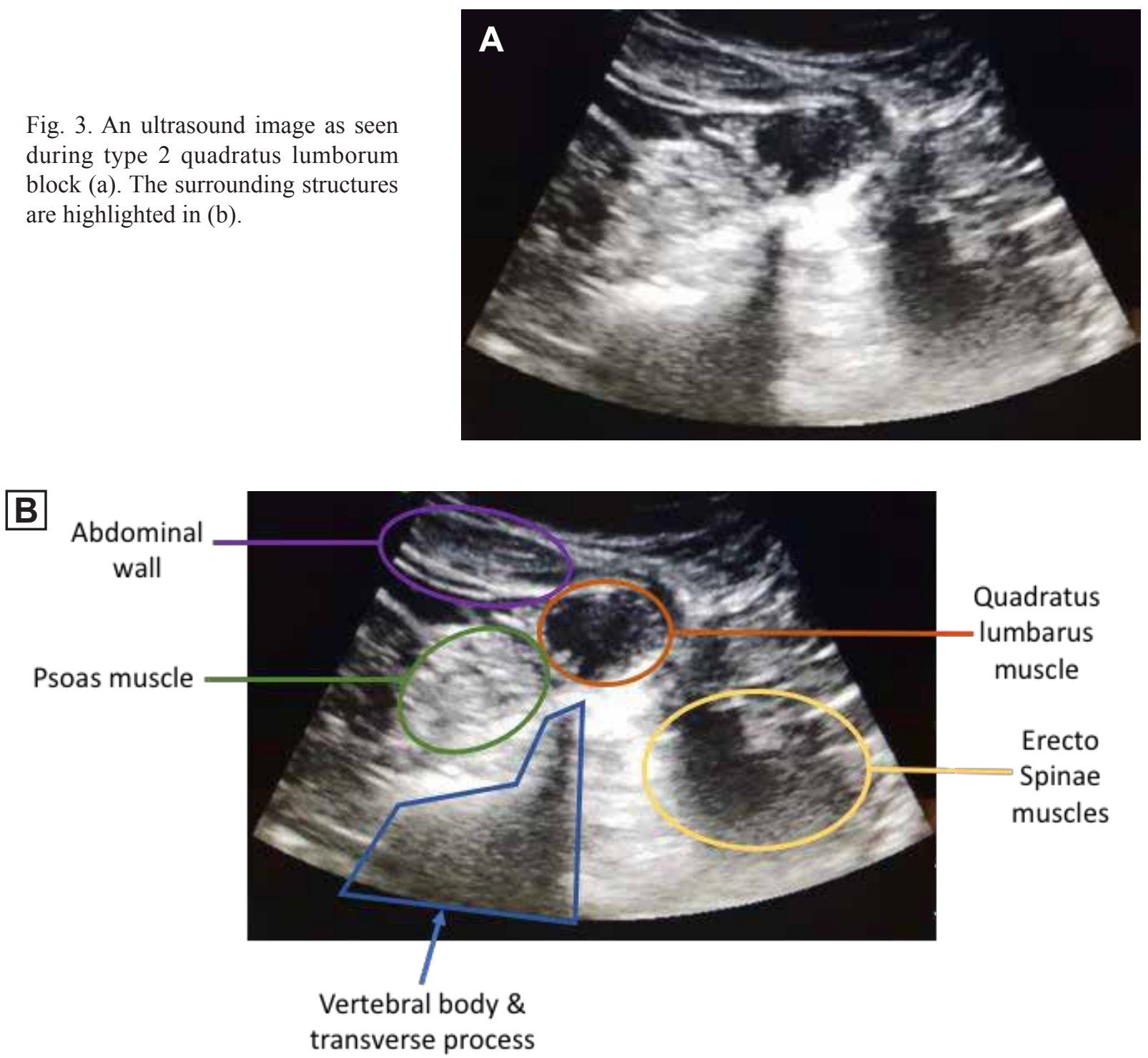

plexus, may further support the notion of myofascial pain playing an important role in these patients.

MPS is a common cause of acute and chronic pain in the general population, most commonly observed as a primary phenomenon. Myofascial pain may also present as a secondary phenomenon, when provoked by such processes as inflammation, infection, or tumors (11).

MPS is frequently diagnosed in patients with cancer pain $(3,4)$, in whom it is thought to be triggered by several mechanisms, including direct muscle invasion by the tumor, muscle irritation by metabolites secreted into the milieu of the tumor, sarcopenia accompanying deconditioning in advanced disease, prolonged bed rest, and by some anticancer treatments such as irradiation and surgery.

QL block could also affect pain indirectly, by spreading of the local anesthetic into nearby tissues via fascial planes. QL block was initially described for the perioperative pain management of abdominal 
surgery, with various authors suggesting an analgesic effect ranging from T7 to L1 (9).

The block is hence likely to encompass the innervation of the abdominal wall. Previous studies have demonstrated an abundance of sympathetic fibers and mechanoreceptors in the TLF, suggesting a role for sympathetic innervation in the pathological process of myofascial syndromes (12). It has been suggested that the injectate spreads into the thoracolumbar plane and into the thoracic paravertebral space (9), and some have claimed that this spread may even extend as far as the spinal nerve roots and the epidural space $(13,14)$, although this claim is debatable.

It should be noted that the observed effect of the block far exceeded the expected action of local anesthetics, as is often the case with chronic pain blocks. This phenomenon could be explained by the local anesthetic breaking a vicious cycle of pain, muscle contraction, and inactivity.

The diagnosis of MPS is based solely on patient interview and physical exam without a need for timeand resource-consuming imaging tests.

QL block is a safe procedure (15) that can be performed at the patient's bed and does not require specialized equipment other than a US machine. According to recent recommendations by the American
Society of Regional Anesthesia and Pain Medicine (16), peripheral musculoskeletal procedures carry low risk of bleeding and may be considered even in anticoagulated patients. Of note, the second patient received the block without complications while being treated with systemic anticoagulation.

\section{Limitations}

This report is limited by the small number of patients and by the lack of a control group. A larger controlled study could confirm the efficacy of the QL block in pancreatic cancer patients.

\section{CONCLUSIONS}

We believe that the cases presented here should prompt clinicians to consider myofascial pain in cancer patients whose pain has not responded completely to standard treatment modalities. Clinicians should probably consider treatment of the MFS and not consider celiac neurolysis a failure if full pain relief was not achieved. In selected patients, simple interventions such as a QL block may help provide some pain relief.

\section{Acknowledgments}

The authors want to thank The Louise and Alan Edwards Foundation for their generous support to the McGill University Health Centre Cancer Pain Program. 


\section{REFERENCES}

1. Stopczynski RE, Normolle DP, Hartman DJ, Haoqiang Ying $\mathrm{H}$, DeBerry JJ, Bielefeldt K, Rhim AD, DePinho RA, Albers KM, Davis BM. Neuroplastic changes occur early in the development of pancreatic ductal adenocarcinoma. Cancer Res 2014; 74:17181727.

2. Epstein JB, Elad S, Eliav E, Jurevic R, Benoliel R. Orofacial pain in cancer: Part II-clinical perspectives and management. J Dent Res 2007; 86:506-518.

3. Hasuo H, Ishihara T, Kanbara K, Fukunaga M. Myofacial trigger points in advanced cancer patients. Indian J Palliat Care 2016; 22:80-84.

4. Vas L, Phanse S, Pai R. A new perspective of neuromyopathy to explain intractable pancreatic cancer pains; Dry needling as an effective adjunct to neurolytic blocks. Indian J Palliat Care 2016; 22:85-93.

5. Eisenberg E, Carr DB, Chalmers TC. Neurolytic celiac plexus block for treatment of cancer pain: A meta-analysis. Anesth Analg 1995; 80:290-295

6. Desai MJ, Saini V, Saini S. Myofascial pain syndrome: A treatment review. Pain Ther 2013; 2:21-36.

7. Willard FH, Vleeming A, Schuenke MD, Danneels L, Schleip R. The thoracolumbar fascia: Anatomy, function and clinical considerations. J Anat 2012; 221:507-536.

8. Cid J, De La Calle JL, López E, Del Pozo C, Perucho A, Acedo MS, Bedmar D, Benito J, De Andrés J, Díaz S, García JA. A modified Delphi survey on the signs and symptoms of low back pain: Indicators for an interventional management approach. Pain Pract 2015; 5:12-21.

9. Ueshima $\mathrm{H}$, Otake $\mathrm{H}$, Lin J. Ultrasound-guided quadratus lumborum block: An updated review of anatomy and techniques.
Biomed Res Int 2017; 2017:2752876.

10. Murouchi T, Iwasaki S, Yamakage M. Quadratus lumborum block: Analgesic effects and chronological ropivacaine concentrations after laparoscopic surgery. Reg Anesth Pain Med 2016; 41:146-150.

11. Giamberardino MA, Affaitati G, Fabrizio A, Costantini R. Myofascial pain syndromes and their evaluation. Best Pract Res Clin Rheumatol 2011; 25:185-198.

12. Tesarz J, Hoheisel U, Wiedenhöfer B, Mense S. Sensory innervation of the thoracolumbar fascia in rats and humans. Neuroscience 2011; 194:302-308.

13. Carline L, McLeod GA, Lamb C. A cadaver study comparing spread of dye and nerve involvement after three different quadratus lumborum blocks. Br J Anaesth 2016; 117:387-394.

14. Adhikary SD, El-Boghdadly K, Nasralah Z, Sarwani N, Nixon $\mathrm{AM}$, Chin KJ. A radiologic and anatomic assessment of injectate spread following transmuscular quadratus lumborum block in cadavers. Anaesthesia 2017; 72:73-79.

15. Abrahams M, Derby R, Horn JL. Update on ultrasound for truncal blocks: A review of the evidence. Reg Anesth Pain Med 2016; 41:275-288.

16. Narouze $\mathrm{S}$, Benzon HT, Provenzano DA, Buvanendran A, De Andres J, Deer TR, Rauck R, Huntoon MA. Interventional spine and pain procedures in patients on antiplatelet and anticoagulant medications: Guidelines from the American Society of Regional Anesthesia and Pain Medicine, the European Society of Regional Anaesthesia and Pain Therapy, the American Academy of Pain Medicine, the International Neuromodulation Society, the North American Neuromodulation Society, and the World Institute of Pain. Reg Anesth Pain Med 2015; 40:182-212. 
IPM Reports 